\title{
A Prospective Study of Etiological Agents Among Febrile Patients in Sierra Leone
}

\author{
Han Wang (D) Jing Zhao · Na Xie - Wanxue Wang · Ruping Qi · \\ Xiaogang Hao · Yan Liu · Stephen Sevalie · Guotao Niu • \\ Yangli Zhang · Ge Wu - Xiaona Lv • Yuhao Chen • Yanfei Ye • \\ Sheng Bi · Moses Moseray · Saidu Cellessy · Ksaidu Kalon • \\ Dawud Ibrahim Baika • Qun Luo
}

Received: April 9, 2021 / Accepted: May 27, 2021 / Published online: June 26, 2021

(C) The Author(s) 2021

\section{ABSTRACT}

Introduction: Sierra Leone has one of the highest burdens of febrile illnesses in the world. As the incidence of malaria diminishes, a better understanding of the spectrum of etiological agents was important for accurate diagnosis and empirical treatment of febrile illness.

Methods: Blood, nasopharyngeal, and fecal specimens were collected from febrile patients for serological, molecular detection, and

Han Wang and Jing Zhao contributed equally to this work.

Supplementary Information The online version contains supplementary material available at https:// doi.org/10.1007/s40121-021-00474-y.

H. Wang $(\bowtie) \cdot$ G. Niu · Y. Zhang · G. Wu · X. Lv .

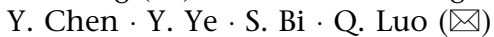

Department of Transfusion Medicine, The Fifth Medical Centre, Chinese PLA (People's Liberation Army) General Hospital, Beijing, People's Republic of China

e-mail: happywanghan@163.com

Q. Luo

e-mail: xienawh@sina.com

J. Zhao

Department of Clinical Diagnostic Centre, The Fifth Medical Centre, Chinese PLA (People's Liberation Army) General Hospital, Beijing, The People's

Republic of China microbiologic culture to identify potential pathogens.

Results: For this prospective study, 142 febrile patients were enrolled. The prevalence of malaria was higher in children aged 5-15 years old $(P=0.185)$ and adults $(P=0.018)$. Acute respiratory infection (ARI) presented more commonly in the under 5 years old group $(P=0.009)$. For diarrhea, all children groups $(P=0.024)$ were predominant. A total of $22.5 \%$ of the febrile patients had malaria infection, $19.7 \%$ had typhoid infection, and $2.8 \%$ were coinfected with malaria and typhoid. ARI was the most common causes of fever, accounting for $31.7 \%$ of patients, influenza A virus, $M y$ coplasma pneumoniae, and five other respiratory pathogens were found. Diarrhea accounted for $16.2 \%$, and seven kinds of diarrhea bacteria were isolated. Hepatitis B accounted for $8.5 \%$,

N. Xie $\cdot$ X. Hao

Department of Inpatient and Medical Record Management, The Fifth Medical Centre, Chinese PLA (People's Liberation Army) General Hospital, Beijing, People's Republic of China

N. Xie

e-mail: xienawh@sina.com

W. Wang

Nursing Department, The Fifth Medical Centre, Chinese PLA (People's Liberation Army) General Hospital, Beijing, People's Republic of China 
including five cases of spontaneous bacterial peritonitis, and ascites smear staining were both Gram-negative bacteria. Tuberculous encephalitis, parasitic diseases (ascaris and filariasis), and skin infection caused by Staphylococcus aureus accounted for 0.7\%, 2.1\%, and $0.7 \%$, respectively.

Conclusions: Evidence of a wide spectrum of febrile etiological agents other than malaria was identified. The spread of malaria rapid diagnostic tests (RDTs) out of hospital and establishment of a national standard for Widal test will reduce the misdiagnosis of febrile diseases. Antibiotics against Gram-negative bacteria are helpful for empirical treatment.

\section{PLAIN LANGUAGE SUMMARY}

Sierra Leone has one of the highest burdens of febrile illnesses in the world. Evidence of a wide spectrum of febrile pathogens other than malaria has been proven in this study. We considered that the etiology of febrile patients was closely related to local geography, heredity, immune features, economic industry, living habits, air pollution, medical and health conditions, and this was fully analyzed and discussed. The screening process used in this study can further simplify and identify the etiological agents of fever in more than $70 \%$ of the study population. This laid the foundation for the establishment of a more simplified and efficient

\section{R. Qi}

Department of Radiology, The Fifth Medical Centre, Chinese PLA (People's Liberation Army) General Hospital, Beijing, The People's Republic of China

\section{Y. Liu}

Department of Clinical Diagnostic Centre, North Hospital District, The Third Hospital of Peking University, Beijing, The People's Republic of China

\section{S. Sevalie}

Headquarters, 34 Military Hospital of Republic of Sierra Leone Armed Forces, Freetown, Sierra Leone

M. Moseray · S. Cellessy · K. Kalon · D. I. Baika Department of Clinical Diagnostic Centre, 34 Military Hospital of Republic of Sierra Leone Armed Forces, Freetown, Sierra Leone diagnosis and treatment process in the local area. We also found the characteristics of age distribution of different febrile diseases. Children were an important susceptible population to fever. This study indicated the importance of reliable diagnostic tests for febrile pathogens and provided the necessary information for RDT requirements. The spread of malaria RDTs out of hospital and establishment of a national standard for Widal test will reduce the misdiagnosis of febrile diseases. For empirical treatment, antimalarial treatment was still targeted at falciparum malaria in Sierra Leone. Antibiotics against Gram-negative bacteria contributed to the empirical treatment of febrile diseases. For patients with acute respiratory tract infection, Gram-positive coccal antibiotics could be candidates for treatment. In addition, systematic and professional treatment of liver diseases should be promoted to reduce infection complications.

Keywords: Fevers; Malaria; Typhoid; Acute respiratory infection; Diarrhea

\section{Key Summary Points}

\section{Why carry out this study?}

Sierra Leone has one of the highest burdens of febrile illnesses in the world. As the incidence of malaria diminishes, a better understanding of the spectrum of etiological agents was important for accurate diagnosis and empirical treatment of febrile illness.

\section{What was learned from the study?}

Evidence of a wide spectrum of febrile pathogens other than malaria has been proven in Sierra Leone. Children were an important susceptible population to fever.

The etiology of febrile patients was closely related to local geography, heredity, immune features, economic industry, living habits, air pollution, medical and health conditions. 
The implementation of malaria RDTs out of hospital and establishment of a national standard for Widal test will reduce the misdiagnosis of febrile diseases.

Antibiotics against Gram-negative bacteria contributed to the empirical treatment of febrile diseases.

\section{DIGITAL FEATURES}

This article is published with digital features, including a summary slide and plain language summary, to facilitate understanding of the article. To view digital features for this article go to https://doi.org/10.6084/m9.figshare. 14680608.

\section{INTRODUCTION}

Fever is the most common symptom of infectious diseases in tropical countries. Western Africa has one of the highest burdens of febrile illnesses, such as malaria, in the world. In Sierra Leone, malaria is the reason behind nearly four in ten hospital consultations [1], with its entire population of over 8 million people at risk of the disease. Substantial progress has been made in fighting malaria. From 2000 to 2015, the incidence rate of malaria cases was reduced by $20-40 \%$ in Sierra Leone [1]. The malaria mortality rates was reduced by more than $40 \%$ between 2010 and 2015, the highest in West Africa [2]. Most notably, in 2013, there were $2,576,550$ suspected malaria cases, with a confirmed rate of $66 \%$. In 2014, there were $2,647,375$ suspected malaria cases, with a confirmed rate of $52 \%$. In $2020,31.1 \%$ of suspected malaria-infected patients were diagnosed with malaria by microscopy and rapid diagnostic tests (RDTs), so the disease in the remaining $68.9 \%$ of patients was caused by other fever-related pathogens $[3,4]$. With the gradual application of malaria RDT in Africa, the proportion of non-malarial febrile illnesses has been found to be increasing, which was particularly noteworthy in malaria endemic areas of Africa [5].
A variety of pathogens were known to cause malaria-like fever, while some viral and bacterial diseases have been identified in febrile patients in Africa [6, 7]. However, it was still difficult to diagnose non-malarial febrile illness in areas with insufficient health resources because of the lack of affordable diagnostic tests and the prevalence of non-malarial febrile illness. These factors lead to misdiagnosis of malaria and delay the optimal treatment of other febrile diseases.

A systematic review of the etiology of febrile illness in low- and middle-income countries shows that only $4.9 \%$ of the case studies come from West Africa [8]. Health workers in Africa believe additional training and point-of-care tests would improve their ability to diagnose and manage non-malarial fevers [9]. Educating caregivers on signs and symptoms of endemic febrile illness, as well as the introduction of additional tests to identify, would be important steps to get patients to appropriate treatment earlier. We therefore did a prospective investigation of the spectrum of etiological agents among febrile patients to improve the understanding of the relative distribution of pathogens such as bacteria, parasites, and viruses, thereby facilitating diagnosis and management decisions for malaria and other febrile diseases.

\section{METHODS}

\section{Study Design and Ethics}

For this prospective study, we recruited consecutive febrile patients seeking healthcare at the 34 Military Hospital of Republic of Sierra Leone Armed Forces and Center for Tropical Infectious Disease Control and Prevention (CTDC) in Freedown from August 2018 through March 2019. The 34 Military Hospital was also the Headquarters of the Joint Medical Unit of Sierra Leone Armed Forces. CTDC was jointly carried out by the Chinese Military Medical Expert Group and the Sierra Leone Military doctors from the 34 Military Hospital. Freetown is the capital and largest city of Sierra Leone, located in the Western Area of the country. The population of Sierra Leone and 
Freetown were 8.05 million and 1.20 million, respectively. Malaria was endemic in the whole of Sierra Leone, and the country's entire population, including Freetown, was at risk of the disease [3].

Patients with continuous fever (axillary temperature $38^{\circ} \mathrm{C}$ or higher), of both sexes, met the following inclusion criteria: first visit due to fever, disease duration of 1 week or less, and not taking antimalarial drugs or antibiotics during the preceding week were included [6]. Infants younger than 2 months of age were excluded, since infectious agents and thus therapeutic approaches can differ substantially according to World Health Organization (WHO) guidelines [10]. The age range of the participants for inclusion in the study was 2-65 years old. Details of the study were discussed with the parents or guardians of children 2-15 years of age; the parents or guardians provided written informed consent. The patients with HIV infection and severe disease symptoms (i.e., neurological diseases, heart disease, shock, trauma, bleeding, etc.) were also excluded from this study. The study was approved by the regional ethics committee in the 34 Military Hospital, Republic of Sierra Leone Armed Forces (Deliberation JMU/1600). The 34 Military Hospital approved this study to carry out studies of etiological agents on human tissues, secretions, body fluids, and blood samples among febrile patients. The study was done according to the Helsinki Declaration. Informed consent was obtained from individuals (or their parents/guardians for children) for participation in this study.

\section{Clinical Assessment and Processing}

For all participants who met the inclusion criteria at the CTDC facility, a baseline questionnaire was administered. Data included demographic data, signs and symptoms, type of medication taken prior to health facility visit, and past infection history. A systematic set of investigations was performed according to the clinical diagnosis and treatment process of fever [11] for all patients. The process was adjusted to three steps to suit the local working conditions:
1. All patients were tested for malaria and typhoid to exclude the two main febrile pathogens. Thus $4 \mathrm{ml}$ of whole blood was collected, $2 \mathrm{ml}$ of which was used for malaria RDT and preparation of blood films for microscopic examination. The rest was used to separate plasma for Widal test.

2. The second step will be to identify those febrile illnesses that are reliably diagnosed on clinical grounds, such as throat pain/tonsillitis, typical skin symptoms including rash/(non-)vesicular rash/blister/ herpes/skin irritation and ulceration/ swelling of the lower leg, meningeal irritation, diarrhea etc.

An acute respiratory infection (ARI) was defined as any acute infection (at most 1 week in duration) that manifested as at least one respiratory sign or symptom and was localized to the upper or lower respiratory tract [12-15]. Upper respiratory infections (URI) were infections of the mouth, nose, throat, larynx, and trachea. URI included nasopharyngitis, sinusitis, pharyngitis, laryngitis, tonsillitis, laryngotracheitis, and so on [16]. Lower respiratory tract infection was a respiratory disease caused by pathogens invading the lower respiratory tract and alveoli, including bronchiolitis and pneumonia [17]. For patients with throat pain/tonsillitis, nasal and throat swabs were collected and immediately placed in tubes containing Universal Transport Medium (Copan, Brescia, Italy). Realtime reverse transcription polymerase chain reaction (PCR) assays for the detection of influenza $A$ and $B$, respiratory syncytial virus, and parainfluenza virus were performed as described previously [18, 19]. Patients with key symptoms or signs (if coughing or with an abnormal chest auscultation) that should lead to specific laboratory investigations will undergo chest X-ray (XH-MD6-32 mobile digital X-ray camera, Shandong, Xinhua Medical Instrument Stock Company Ltd, China). Sputum culture, serologies (IgM) for Mycoplasma pneumoniae and Chlamydia pneumoniae were also performed.

Patients with skin symptoms suspected of 
herpes simplex virus, mumps, rubella, measles, varicella zoster virus, dengue fever, and filariasis will undergo venous puncture $(4 \mathrm{ml})$ for serological IgM detection. Skin secretion samples need to be identified by microbiological culture. Cerebrospinal fluid (CSF) test was performed in patients with meningeal irritation syndrome.

Patients with typical diarrhea symptoms need to collect stool samples for identification by microbial culture and direct microscopic examination.

3. Patients with history or suspected liver disease, abnormal alanine amino transferase (ALT) or ascites were serological screened for hepatitis A, B, C, and E. If accompanied by renal dysfunction, hantavirus was also screened for. Ascites routine test was performed in all patients with ascites.

\section{Laboratory Analyses}

\section{Malaria}

Positive malaria RDT [malaria Ag Plasmodium falciparum (P. falciparum)/Plasmodium vivax (P. vivax), SD BIOLINE, USA] OR positive blood slide for malaria, regardless of the presence of another cause of fever. Malaria microscopy: Thick films stained with $10 \%$ Giemsa were used for definitive parasite counts; 200 high power fields were screened before a slide was declared negative.

\section{Typhoid}

Positive typhoid RDT OR positive stool culture Salmonella typhi (S. typhi).

Serological diagnosis was carried out using Widal agglutination test (Tianrun biopharmaceutical Co., Ltd, Ningbo, China) to determine the antibody titers of the sera against Salmonella $\mathrm{H}$ (flagella) and $\mathrm{O}$ (somatic) antigens. The serological testing was done in accordance with manufacturers' guidelines, an antibody titer of at least 1:80 for both typhi $\mathrm{O}$ and $\mathrm{H}$ was taken as a cutoff value to indicate recent infection of typhoid fever.

\section{Microbiologic Investigations}

Stool samples were collected in clean widemouthed plastic containers from 23 patients with diarrhea. All stool samples were macroscopically observed for areas that contain blood and/or mucus/parasite. A loopful of each stool sample was inoculated onto Salmonella-Shigella agar (SSA) and MacConkey agar (MAC, all from ProSpecT, Remel, Lenexa, Kansas, USA). The inoculated plates were then incubated at $37^{\circ} \mathrm{C}$ for $24 \mathrm{~h}$. Isolates identified as $S$. typhi were differentiated from other Salmonella spp. using Triple Sugar Iron (TSI) agar (Becton Dickinson, Franklin Lakes, USA) [20]. Cultural and morphological characteristics of the isolates were studied thereafter and the isolates were duly identified following standard microbiological methods using VITEK 2 compact automatic bacteria analyzer (bioMérieux Company, France) [21-23]

Sputum specimens were treated with equal volume of sputasol, vortexed for $1 \mathrm{~min}$, and then held for $30 \mathrm{~min}$ at room temperature; 10 $\mu \mathrm{L}$ of digested sample was dispensed onto each of three different plates and streaked in a threequadrant pattern with an inoculation loop. The media used in this study were Columbia agar with 5\% sheep blood (blood agar, Oxoid, England), chocolate agar (Choc agar, Oxoid, England), and China blue agar (a strong selective medium which is similar to MacConkey agar plate; CB agar, Beiruite Biotechnology Co., Ltd, Zhengzhou, China). Skin secretion samples were dispensed onto each of those three different plates. Sputasol (Oxoid, England) was used to digest the sputum specimens [24].

\section{Serological Test}

Serologies were performed using the following commercial kits: IgM for mumps, varicella zoster virus, and hantavirus: Beijing Beier Bioengineering Co., Ltd, Beijing, China; IgM for herpes simplex virus 1 and 2, measles, rubella, M. pneumoniae, C.pneumoniae, dengue fever: Oumeng medical diagnosis Co., Ltd, Beijing, China; IgM for HAV: WANTAI BioPharm Co., Ltd, Beijing, China. Hepatitis B markers (HBsAg, 
anti-HBS, HBeAg, anti-HBE, anti-HBC) and IgG for Mycobacterium tuberculosis (TB): Aibo biomedical Co., Ltd, Hangzhou, China; IgM for HCV: InTec PRODUCTS, INC. Xiamen, China; $\operatorname{lgM} / \operatorname{lgG}$ for HEV: Beijing modern gold biology technology Co., Ltd, Beijing, China; IgM for filariasis: BinaxNOW Filariasis, Inverness Medical, Portland, ME, USA, respectively. The above operations and results are judged according to the manufacturers' instructions.

\section{Ascitic Fluid and Cerebrospinal Fluid (CSF) Test}

(1) Routine test $[25,26]$ and (2) biochemistry test were carried out by an automatic biochemical analyzer (TC6030L, TECOM, Jiang Xi, China). Ascitic fluid infection was classified according to the criteria described by Wiest et al. [25]. Meningitis was defined as previously described [26]. Ascites and cerebrospinal fluid were centrifuged and precipitated immediately after extraction. The precipitates were smeared and stained with Gram stain.

\section{Statistical Analyses}

Demographic characteristics were summarized in terms of means and standard deviation or percentages. The distribution of clinical diagnosis of 142 patients with fever was represented by pie chart. We compared the distribution of different age groups of patients with major diseases, using the chi-square test. $P$ values of less than 0.05 were considered statistically significant. The SPSS 17.0 software package (SPSS, Chicago, IL, USA) was used for statistical analysis.

\section{RESULTS}

\section{Clinical and Household Characteristics of Study Participants}

A total of 158 febrile patients were initially enrolled; 16 were subsequently excluded from the study ( 2 were diagnosed with liver cancer, 12 with HIV, 1 with humeral fracture, and 1 with suspected hereditary disease due to abnormal hemoglobin electrophoresis). Of the 142 patients enrolled in the study, the average temperature at enrollment was $38.4 \pm 1.3^{\circ} \mathrm{C}$. Women accounted for $51.2 \%$ of the total. Among the major diseases, the female infection rate was the highest in malaria $(62.5 \%)$, followed by typhoid $(53.6 \%)$, diarrhea $(47.8 \%)$, and ARI (46.7\%). For all patients combined, the duration of fever before admission was 5.7 (mean) and 1.8 (median) days. Of the symptoms commonly found in febrile patients, chill $(41.5 \%)$, fatigue $(27.5 \%)$, headache $(26.1 \%)$,

Table 1 Clinical characteristics of the study participants at enrollment

\begin{tabular}{|c|c|}
\hline Variable & Value $^{\mathrm{a}} N(\%)$ \\
\hline Age, years (range) & $24.5(2-65)$ \\
\hline$<5$ & $31(21.8 \%)$ \\
\hline $5-15$ & $34(23.9 \%)$ \\
\hline $15-55$ & $55(38.7 \%)$ \\
\hline$>55$ & $22(15.5 \%)$ \\
\hline \multicolumn{2}{|l|}{ Sex } \\
\hline Number of male patients & $68(47.9 \%)$ \\
\hline Number of female patients & $74(52.1 \%)$ \\
\hline \multicolumn{2}{|l|}{ Chief symptom } \\
\hline Mean temperature $\left({ }^{\circ} \mathrm{C} \pm \mathrm{SD}\right)$ & $38.4( \pm 1.3)$ \\
\hline Chill & $59(41.5 \%)$ \\
\hline Fatigue & $39(27.5 \%)$ \\
\hline Headache & $37(26.1 \%)$ \\
\hline Abdominal pain & $28(19.7 \%)$ \\
\hline Cough & $25(17.6 \%)$ \\
\hline Diarrhoea/dysentery & $23(16.2 \%)$ \\
\hline Nausea & $21(14.8 \%)$ \\
\hline Stomach pain & $15(10.6 \%)$ \\
\hline Vomiting & $14(9.9 \%)$ \\
\hline
\end{tabular}


and abdominal pain (19.7\%) were common (Table 1).

Among the patients, the average age was $24.5 \pm 24.8$ years. The patients were divided into four groups according to age: $<5$ years old, 5-15 years old, $15-55$ years old (adult), and $>55$ years old (elderly group) (Table 1 ). Of the 142 patients, $31(21.8 \%)$ were $<5$ years old, 34 (23.9\%) were $5-15$ years old, 55 (38.7\%) were $15-55$ years old, and $22(15.5 \%)$ were $>55$ years old [27]. The differences in the distribution of the age group were statistically significant $\left(\chi^{2}\right.$ values $=16.732, \quad P=0.021)$. Malaria, typhoid fever, diarrhea, and ARI were the major diseases found in this study, accounting for more than $90 \%$ of the total. The prevalence of malaria was higher in children aged 5-15 years old $\left(\chi^{2}=9.549, P=0.185\right)$ and adults $\left(\chi^{2}=18.852\right.$, $P=0.018)$, as expected in malaria endemic areas [28]. This epidemic trend was basically similar to typhoid fever and diarrhea, but not exactly the same as diarrhea. Diarrhea was predominant in children $\left(\chi^{2}=15.358, P=0.024\right)$, with a total proportion of $60 \%$ higher than adults and the elderly. ARI occurred in all age groups, but presented more commonly in $<5$ years old $\left(\chi^{2}=22.165, P=0.009\right)$. Children $(<15$ years old) are the important population with febrile diseases (45.8\%) (Figs. 1, 2).

\section{Diagnoses}

The distribution of clinical diagnoses of all 142 febrile patients is shown in Table 2. Figure 2 shows a flowchart of investigations applied.

A total of $22.5 \%$ of the febrile patients had malaria infection, $19.7 \%$ had typhoid infection, $31.7 \%$ had ARI, $16.2 \%$ had diarrhea, $8.5 \%$ had hepatitis B infection, $2.8 \%$ had typical skin symptom, $2.10 \%$ had parasitic diseases, and

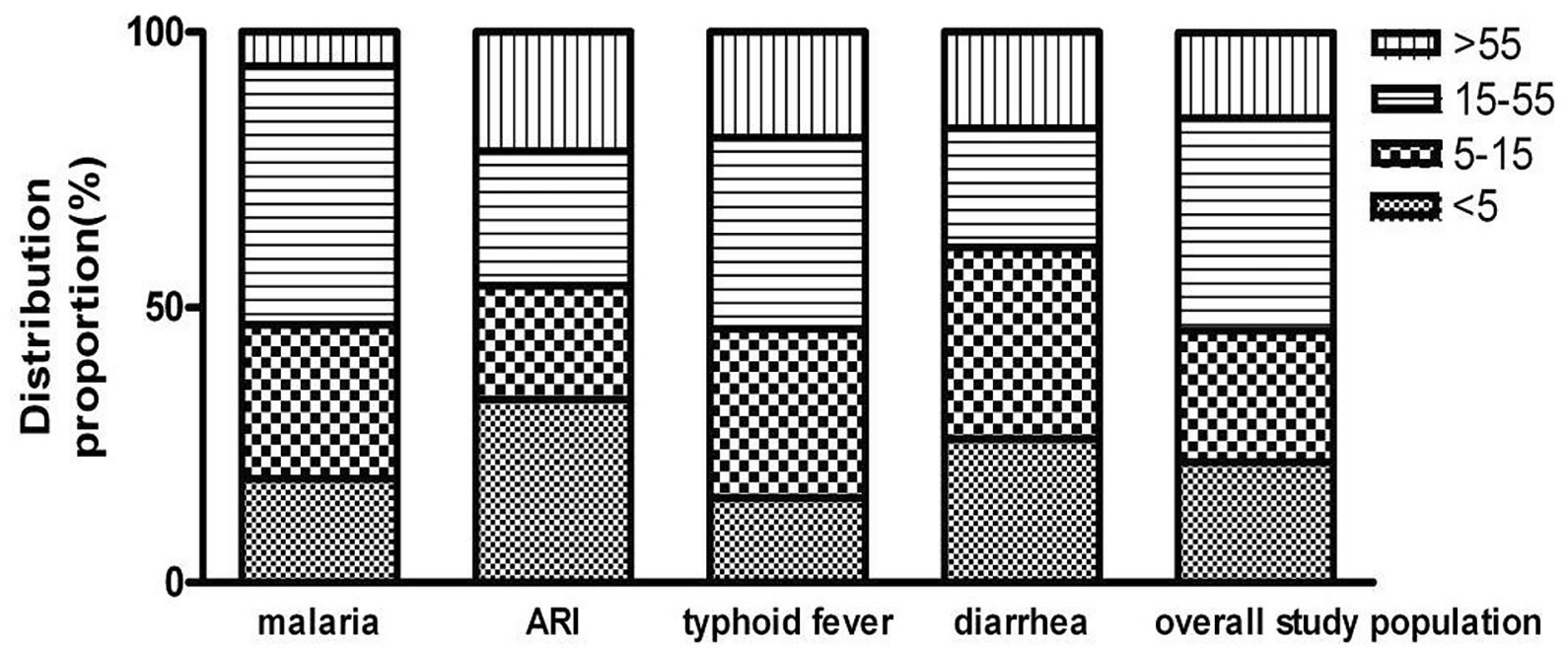

Fig. 1 The distribution of major diseases according to age group. The patients with major diseases were divided into four groups: $<5$ years old, 5-15 years old, 15-55 years old (adult) , and $>55$ years old (elderly group) [27]. Of the 32 malaria patients, $6(18.8 \%)$ were $<5$ years old, $9(28.1 \%)$ were $5-15$ years old, 15 (46.9\%) were $15-55$ years old, and 2 cases $(6.3 \%)$ were $>55$ years old. 45 cases of acute respiratory infection, the distribution of the above four age groups: 15 cases (33.3\%), 9 cases (20.0\%), 11 cases (24.4\%), and 10 cases $(22.2 \%)$. The distribution of 28 cases of typhoid fever: 4 cases (15.4\%), 8 cases (30.8\%), 9 cases (34.6\%), and 5 cases (19.2\%). The distribution of 23 cases of diarrhea: 6 cases (26.1\%), 8 cases (34.8\%), 5 cases (21.7\%), and 4 cases $(17.4 \%)$, respectively. The chi-square test was used to compare the distribution of the age index. The differences in the distribution of the age group were statistically significant $\left(\chi^{2}=16.732, P=0.021\right)$. Briefly, malaria was predominant in children aged $5-15$ years old $\left(\chi^{2}=9.549, \quad P=0.185\right)$ and adults $\left(\chi^{2}=18.852\right.$, $P=0.018)$. Diarrhea was predominant in children $\left(\chi^{2}=15.358, P=0.024\right)$, with a total proportion of $60 \%$ higher than adults and the elderly. Acute respiratory infection presented more commonly in $<5$ years old $\left(\chi^{2}=22.165, P=0.009\right)$ 


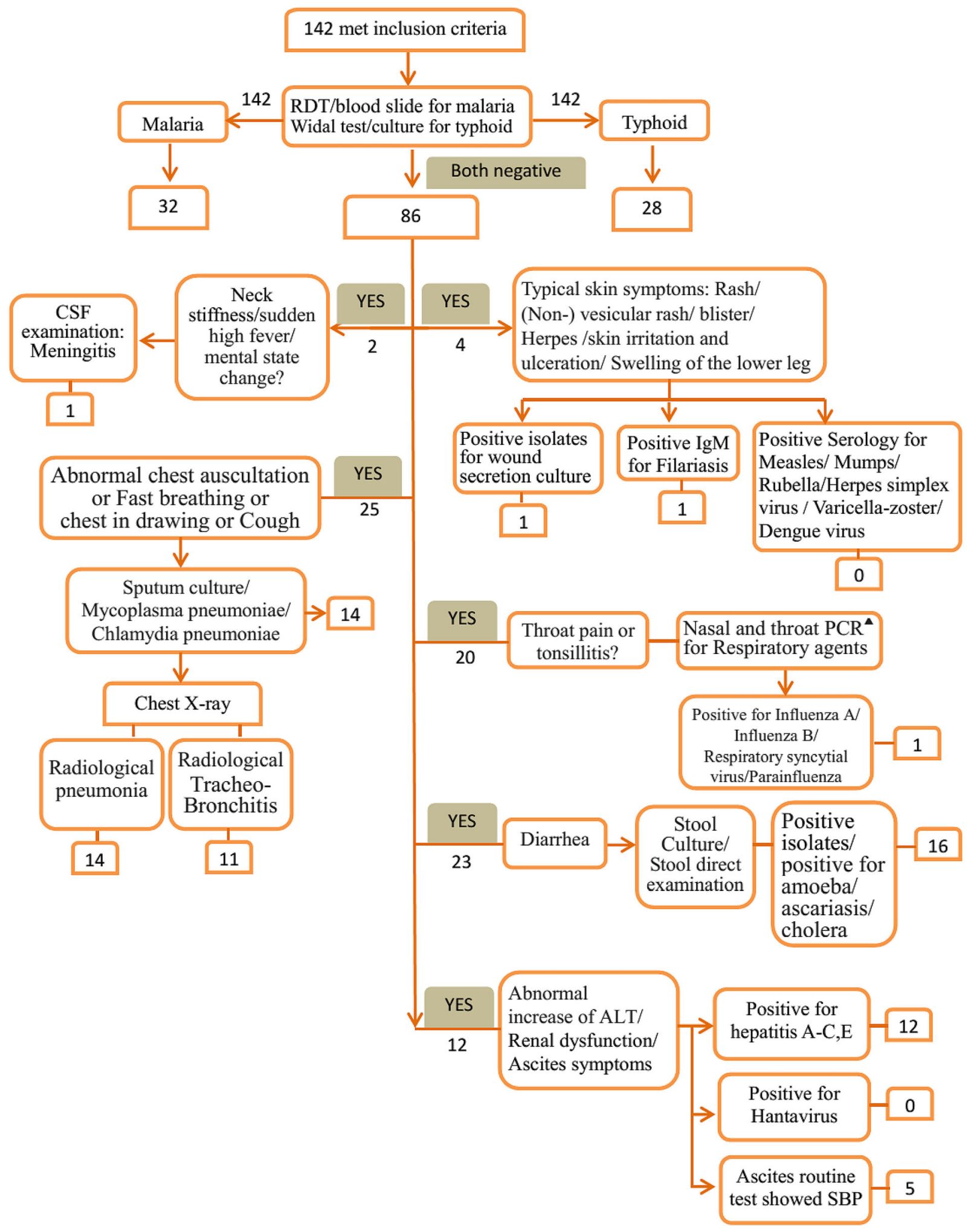


4Fig. 2 Flowchart of investigations performed in 142 febrile patients. $P C R$ polymerase chain reaction, $R D T$ rapid diagnostic test, $C S F$ cerebrospinal fluid, $A L T$ alanine aminotransferase, $A b$ antibody, $S B P$ spontaneous bacterial peritonitis. ${ }^{\boldsymbol{\Lambda}} \mathrm{Nasal}$ and throat PCR for respiratory agents: influenza $A$ and $B$, respiratory syncytial virus, and parainfluenza

$1.40 \%$ had meningismus (Fig. 3). Each individual pathogen is listed in Table 2 .

\section{Prevalence of Malaria and Typhoid Fever}

All patients were tested and screened for malaria and typhoid. Thirty-two patients $(22.5 \%)$ were diagnosed with malaria infection. Of them, 30 cases $(21.1 \%)$ were positive for $P$. falciparum, 1 case $(0.7 \%)$ for $P$. vivax, and $1(0.7 \%)$ for both $P$. falciparum and $P$. vivax. Twenty-eight (19.7\%) patients had antibody titers of at least 1:80 for both $\mathrm{O}$ and $\mathrm{H}$ antigens; of them, 20 (14.1\%) had at least 1:320 titers (Supplementary Table 1). We collected 14 stool samples from patients with typhoid fever, but only two strains of $S$. typhi were isolated (Table 2). The Widal test result showed that the prevalence of coinfection was 4 $(2.8 \%)$. All four of them (100\%) were coinfected with $P$. falciparum; no cases were with $P$. vivax. Using stool culture, the prevalence of coinfection was only $1(0.7 \%)$.

\section{Prevalence of Diarrhea}

Stool culture was performed with samples from 23 patients (16.2\%) with diarrhea. A total of 55 stool samples were collected, and 14 positive isolates $(9.9 \%)$ were obtained. Non-typhoidal serovars of Salmonella enterica (NTS) were the most common isolate with four positive cases $(28.6 \%)$. In addition to NTS, a variety of diarrhea bacteria were isolated, including Shigella flexneri in three cases (21.4\%), enteropathogenic Escherichia coli in two cases (14.3\%), Shigella sonnei in one case (7.1\%), and Staphylococcus aureus in one case $(7.1 \%)$. Two cases $(14.3 \%)$ of Vibrio parahaemolyticus and one case $(7.1 \%)$ of Aeromonas hydrophila were also isolated from feces. Stool routine examination showed that two patients were infected with Ascaris lumbricoides; no Amoeba histolytica was found.

\section{Prevalence of Acute Respiratory Infection}

Acute respiratory infection was the most common cause of fever, accounting for 45 of 142 patients $(31.7 \%)$ in the study. Infections were localized to the upper respiratory tract in 20 patients (including 9 cases of rhinitis, 7 cases of tonsillitis, and 4 cases of pharyngitis) and to the lower respiratory tract in 25 patients ( 11 with bronchiolitis and 14 with radiologically confirmed pneumonia). Twenty patients with upper respiratory tract were screened for influenza virus A, influenza B, parainfluenza, and respiratory syncytial virus by reverse transcription PCR. We found one patient was positive for influenza A virus, while the other three viruses were all negative in patients with upper respiratory tract infection. Sputum samples were collected from 18 patients with lower respiratory tract infection. Sputum culture showed that there were four cases of Pseudomonas aeruginosa, three cases of Klebsiella pneumoniae, three cases of E. coli, two cases of S. aureus, and one case of S. pneumoniae. Gram-negative rods accounted for $76.9 \%$ of the total. In addition, $\operatorname{lgM}$ antibody of M. pneumoniae was positive in one patient.

\section{Prevalence of Other Common Communicable Diseases}

A total of 12 patients with fever were diagnosed as hepatitis B infection, including nine cases (75\%) of cirrhosis with ascites. Ascites test showed that five cases were spontaneous bacterial peritonitis (SBP), and all of them were Gram-negative bacteria by smear staining. The mean value of white blood cell count in ascites of patients with SBP was $4.7 \times 10^{9}$ cells/L, and the mean value of polymorphonuclear leukocyte count was $3.8 \times 10^{9}$ cells/L. CSF examination was performed in one patient with tuberculosis, and the results were consistent with the characteristics of tuberculous encephalitis [26]. Parasitic diseases were still prevalent. Except for malaria and A. lumbricoides found in feces, serological test showed that one patient was positive for filariasis IgM antibody. One case had obvious suppurative infection of 
Table 2 Number and proportion of the study participants at enrollment by each individual pathogen

\begin{tabular}{|c|c|c|c|}
\hline Pathogen & Specimen tested & Method of detection & $\begin{array}{l}\text { Number (\%) of infected } \\
\text { Total } n=142\end{array}$ \\
\hline \multicolumn{4}{|l|}{ Bacteria } \\
\hline Pseudomonas aeruginosa & Sputum & Culture & $4(2.8 \%)$ \\
\hline Klebsiella pneumoniae & Sputum & Culture & $3(2.1 \%)$ \\
\hline Escherichia coli & Sputum & Culture & $3(2.1 \%)$ \\
\hline Staphylococcus aureus & Sputum & Culture & $2(1.4 \%)$ \\
\hline Streptococcus pneumoniae & Sputum & Culture & $1(0.7 \%)$ \\
\hline Mycoplasma pneumoniae & Serum & Serology & $1(0.7 \%)$ \\
\hline Chlamydia pneumoniae & Serum & Serology & $0(0.0 \%)$ \\
\hline Staphylococcus aureus & Wound secretion & Culture & $1(0.7 \%)$ \\
\hline Salmonella enterica & Stool & Culture & $4(2.8 \%)$ \\
\hline Shigella flexneri & Stool & Culture & $3(2.1 \%)$ \\
\hline Enteropathogenic E. coli & Stool & Culture & $2(1.4 \%)$ \\
\hline Shigella sonnei & Stool & Culture & $1(0.7 \%)$ \\
\hline Vibrio parahaemolyticus & Stool & Culture & $2(1.4 \%)$ \\
\hline Aeromonas hydrophila & Stool & Culture & $1(0.7 \%)$ \\
\hline Staphylococcus aureus & Stool & Culture & $1(0.7 \%)$ \\
\hline Cholera $O 1$ & Stool & Microscopy & $0(0.0 \%)$ \\
\hline Cholera 0139 & Stool & Microscopy & $0(0.0 \%)$ \\
\hline \multicolumn{4}{|l|}{ Viruses } \\
\hline Influenza A & NP & RT-PCR & $1(0.7 \%)$ \\
\hline Influenza B & NP & RT-PCR & $0(0.0 \%)$ \\
\hline Respiratory syncytial virus & $\mathrm{NP}$ & RT-PCR & $0(0.0 \%)$ \\
\hline Parainfluenza & $\mathrm{NP}$ & RT-PCR & $0(0.0 \%)$ \\
\hline Herpes simplex virus & Serum & Serology & $0(0.0 \%)$ \\
\hline Mumps & Serum & Serology & $0(0.0 \%)$ \\
\hline Measles & Serum & Serology & $0(0.0 \%)$ \\
\hline Varicella zoster virus & Serum & Serology & $0(0.0 \%)$ \\
\hline Rubella & Serum & Serology & $0(0.0 \%)$ \\
\hline Dengue & Serum & Serology & $0(0.0 \%)$ \\
\hline Hepatitis A & Serum & Serology & $0(0.0 \%)$ \\
\hline Hepatitis B & Serum & Serology & $12(8.5 \%)$ \\
\hline Hepatitis C & Serum & Serology & $0(0.0 \%)$ \\
\hline
\end{tabular}


Table 2 continued

\begin{tabular}{llll}
\hline Pathogen & Specimen tested & Method of detection & $\begin{array}{l}\text { Number (\%) of } \\
\text { infectedTotal } \boldsymbol{n}=\mathbf{1 4 2}\end{array}$ \\
\hline Hepatitis E & Serum & Serology & $0(0.0 \%)$ \\
Hantavirus & Serum & Serology & $0(0.0 \%)$ \\
Parasites & & & \\
Plasmodium falciparum & Blood & Antigen detection and microscopy & $30(21.1 \%)$ \\
$\begin{array}{l}\text { Plasmodium vivax } \\
\text { P. falciparum and P. vivax }\end{array}$ & Blood & Antigen detection and microscopy & $1(0.7 \%)$ \\
Typhoid & Blood & Antigen detection and microscopy & $1(0.7 \%)$ \\
Ascariasis & Stool & Serology & $28(19.7 \%)$ \\
Filariasis & Blood & Microscopy & $2(1.4 \%)$ \\
Amoeba histolytica & Stool & Merology & $1(0.7 \%)$ \\
Others & & & $0(0.0 \%)$ \\
$\mathrm{G}^{+} / \mathrm{G}^{-}$induced peritonitis & Ascites & Microscopy & \\
Tubercle bacillus & CSF & Microscopy & $5(3.5 \%)$ \\
\hline
\end{tabular}

$N P$ nasopharynx, $R T$-PCR reverse transcription polymerase chain reaction, $G$ Gram-positive bacteria, CSF cerebrospinal fluid

skin and mucosa. S. aureus was isolated from the skin secretion of the patient.

No Cholera O1/O139, A. histolytica, herpes simplex virus, mumps, measles, rubella, varicella zoster virus, dengue, hepatitis A, hepatitis $C$, hepatitis $E$, or hantavirus was found in the 142 febrile patients by means of the applied detection methods (Table 2).

\section{DISCUSSION}

In this study, the screening process used was able to identify more than $70 \%$ of the fever pathogens. More pathogens will be found if respiratory viruses are screened more widely. This process laid the foundation for the establishment of a more simple and feasible clinical evaluation process of febrile disease in Sierra Leone. Our findings also indicated that although malaria and typhoid fever were still serious (39.4\% in total), ARI and diarrhea have became the leading causes of febrile illness, reaching $47.9 \%$. In addition, hepatitis B and parasite were also important etiological agents that can not be ignored.

Sierra Leone continued to have high child mortality rates, with the United Nations estimating that under 5 mortality rate (U5MR) was $114 \%$ [29]. This study confirmed that not only children under 5 years old but also children under 15-year-old children were important susceptible populations to fever. The incidence rate of malaria was related to the frequency of malaria exposure, while younger children may have longer protection in tents. Therefore, malaria was predominant in children aged 5-15 years and adults, and its prevalence generally increased with age. The risk of diarrheal pathogens such as NTS was higher in children 


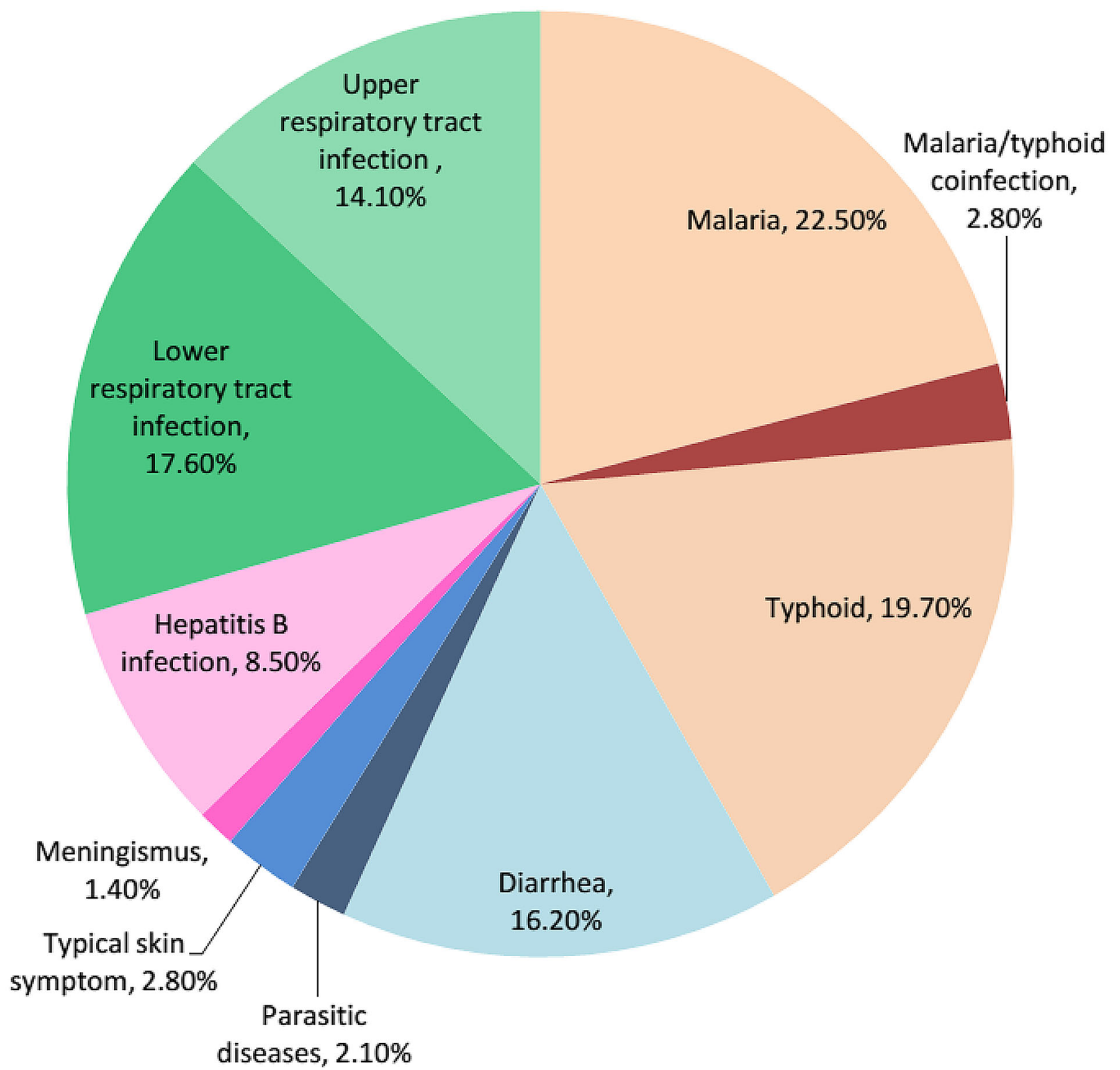

Fig. 3 Distribution of clinical diagnoses among 142 febrile patients. Numbers are percentages of all diagnoses. The distribution of clinical diagnoses of febrile illness was as follows: 32 cases of malaria, 28 of typhoid, 23 of diarrhea, 20 of upper respiratory tract infection, 25 of lower respiratory tract infection, 12 of hepatitis $B$ infection, 4 of

$[30,31]$, and the risk factors involved were malaria, malnutrition, sickle cell disease, HIV, and anemia [28]. Children accounted for more than $60 \%$ of the diarrhea group in the current study. ARI was a global problem and was generally susceptible in all age groups, but it typical skin symptom, 3 of parasitic diseases, and 2 of meningismus. The parasitic diseases cases were from 2 patients with diarrhea and 1 patient with typical skin symptoms due to swelling of lower leg. Percentages may not sum to 100 because of coinfection and rounding

seemed to be more common in younger children because of weak immunity. In terms of children accounting for $45.8 \%$ of febrile patients, early antimalarial and antibiotic treatment was helpful to reduce U5MR and improve children's health. 
In this study, $22.5 \%$ of the febrile patients had malaria infection, which was consistent with a recent malaria detection study [32]. Malaria in the country once reached $40 \%$ of outpatient morbidity [1]. Owing to the implementation of the National Malaria Control Programme, the malaria infection rate has shown a downward trend, and people's awareness of timely antimalaria drugs for infection has been significantly improved. In some African countries, in the case of treatment, buying medicine from pharmacy shops was common $(48 \%)$. This was followed by the use of herbal remedies (31\%), while appreciable numbers adopted self-treatment methods (18\%) [33]. Furthermore, in endemic settings, less than half of patients carried malaria parasite in their blood without showing any symptoms. This asymptomatic parasitemia could be attributed to the level of immunity they have developed because of constant mosquito bites and frequent attack of malaria infection [33]. These factors reduced the number of patients with malaria who have access to health facilities. In addition, women seemed to be more vulnerable to malaria infection (62.5\%). Our hospital provided important medical services for residents in the capital and the surrounding rural areas. In Sierra Leone, malaria prevalence in rural areas was significantly higher than that in urban areas [1]. When we visited rural areas, we found that most men left rural areas to work in the capital city, which was also the earliest and most frequent area in the country where malaria programs were implemented [1]. As a result, women may have more opportunities to be infected with malaria when they were farming. Owing to the high sensitivity of malaria RDT, it was suitable for screening. Therefore, the spread of malaria RDTs outside the hospital, such as pharmacy and chief tribe, could help reduce the misdiagnosis rate.

$P$. falciparum was the dominant species in the epidemic area, accounting for 93.8\%, while $P$. vivax only accounted for $6.2 \%$. Unlike earlier reports, we did not find Plasmodium ovale [34]. $P$. vivax had traditionally shown a small prevalence in West and Central Africa, attributed to the high prevalence of Duffy-negative people [Fy(a-b-)] who were described as being resistant to P. vivax infection [35]. The prevalence of $P$. vivax was significantly lower than that in Ethiopia, East Africa, where the infection rate of vivax malaria could reach $56.2 \%$ [36]. Furthermore, earlier studies have identified Anopheles gambiae, Anopheles funestus, Anopheles melas, and Anopheles nili as efficient local malaria vectors, with $P$.falciparum being the dominant parasite species [37, 38]. Therefore, antimalarial treatment was still aimed at falciparum malaria.

The Widal test was the main method for typhoid detection available in most medical facilities in Sierra Leone, microbiologic culture was still relatively lacking [39]. The prevalence of typhoid fever by Widal test was $19.7 \%$, which was much lower than the report of $31.4 \%$ in Bo, another city in Sierra Leone [40]. It was also lower than a more recent study wherein $44.9 \%$ of the cases in Freetown had antibody titer of at least 1:80 for serotype $O$ and $H$ [41]. Similarly, we noted that in Nigeria, the prevalence of typhoid varies significantly from $10.3 \%$ to $73.9 \%$ in different cities and regions [36]. Repeated exposure to $S$. typhi in endemic areas made it difficult to establish a stable baseline titer for the population. A cutoff titer of $\mathrm{O}$ agglutinin of $1: 40,80,100,160$, and 200 , while that of $\mathrm{H}$ agglutinin was $1: 40,80,160$, and 200 respectively, was the optimal indicator of typhoid fever in different regions [42-46]. Through the preliminary study on the titer of the Widal test in local healthy physical examination populations, we found that $1: 160$ was more suitable as a positive threshold, and at most 1:80 may be the baseline titer of the population, similar to the study of Egypt in Africa (Supplementary Table 1) [45]. We found that most patients $(71.4 \%)$ had high titer (at least 1:320) of Widal test. There were only three patients with typhoid fever whose titer was $1: 80$. Typhoid was the only cause of fever found in these three patients and we immediately treated them with antibiotics. Although from the perspective of therapeutic diagnosis, the rehabilitation of patients could be used as the basis for the diagnosis of typhoid infection, it still could not rule out the possibility of other bacterial or even viral infections. In addition, according to a survey, $32 \%$ of patients would delay 4-6 days or more before seeking treatment 
until the symptoms were obvious [33]. Toward the end of the first week of illness, titers of either $\mathrm{O}$ or $\mathrm{H}$ antibody may rise to as high as 1:160 [45, 47, 48]. Accordingly, the current study also indicated that as a high epidemic area of typhoid fever, appropriately increasing the cutoff value to 1:160 may be a better choice, which could narrow the scope of gray area and reduce the false positive results. It difficult to provide reliable diagnostic results with the Widal test was because of its variability and cross-reactivity [49]. The baseline of antibody titer and the standardization were the key issues affecting the Widal test. Therefore, national standards can be established to improve the detection accuracy and effectively reduce the detection variation between regions.

Even in a high titer in Widal test, the causative organism may be due to other species of Salmonella, rather than S.typhi. Sansone and Hennekens published a case report where the Widal reaction to typhoid $\mathrm{O}$ antigen on admission for an unexposed patient was 1:320, with an increase in titer to $1: 20,480$ by the fourth day [50]. In this study, the result of malaria and typhoid coinfection was $2.8 \%$, lower than the previous $6.5 \%$ reported in Ethiopia [36], and comparable with the 2.5\% reported in Cameroon [51]. Some coinfection rates could reach $17 \%$ or higher, which was overestimated because of the coexistence of S. typhimurium [52]. Definitive diagnosis of typhoid required isolation of $S$. typhi from blood or stool [53]. However, only two cases of $S$. typhi were isolated from feces. It was reported that the positive rate of $S$. typhi in blood/feces culture was only 2.6-8.9\% [54]. High-quality microbiologic diagnostics may not always be available. In addition, it was difficult to obtain fecal samples from every Widal test-positive outpatient. However, if a simple manual microbial identification method can be used to identify diarrhea bacteria, such as S. typhimurium, it can play an important role in the differential diagnosis.

Diarrhea was an important cause of fever. Among the 14 positive isolates, NTS was the most common isolate $(28.6 \%)$. This was consistent with the previous report that NTS predominated in all African regions, especially sub-
Saharan Africa [28]. In malaria endemic areas, the rate of NTS increased during the high transmission/rainy season, which was related to the antibody production induced by malaria, mucosal barrier dysfunction, hemolysis, and so on [28]. The rainy season also had a direct impact on diarrhea, while in Sierra Leone it accounted for half of the year. Improper treatment of human excreta would lead to the rapid spread of diarrhea bacteria. In addition to NTS, a variety of diarrhea bacteria were isolated, including S. flexneri, S. sonnei, enteropathogenic E. coli, and $S$. aureus, which were similar to the diarrhea spectrum in South Africa [55].

Sierra Leone is adjacent to the Atlantic Ocean and has rich fishery resources. About 95\% of people in Sierra Leone depend on crude fuels, mainly wood and charcoal [56, 57]. Inadequate combustion leads to seafood not being fully cooked and causes diarrhea. Diarrhea pathogens associated with fishery and aquatic products, including $V$. parahaemolyticus and A. hydrophila, were isolated. In 2012, the country had an outbreak of cholera [58]. Therefore, considering the sample size of the study, these isolated strains were present as relatively minor but potentially significant pathogens. Antibiotics against Gram-negative bacteria contributed to the empirical treatment of diarrhea. Patients should be reminded to fully cook their food and vinegar can be added to seafood.

Acute respiratory infection was the most common cause of fever, accounting for $31.7 \%$. Although only one of our patients tested positive for influenza A virus, the other three viruses were all negative in patients with upper respiratory tract. Therefore, it may suggest that upper respiratory infection is mainly caused by rhinovirus, adenovirus, or coronavirus $[6,16]$. More than $75 \%$ of the pathogens causing lower respiratory tract infection were Gram-negative rods, including $P$. aeruginosa, K. pneumoniae, and E. coli. We also isolated Streptococcus pneumoniae and S.aureus, which may well be one of the major triggers for the development of severe pneumonia [6]. This spectrum of isolates was similar to that of Gambia in West Africa [59]. Antibiotics for Gram-negative bacilli and Gram- 
positive cocci can be used as prior empirical treatment for patients with ARI.

Risk factors for ARI include crowded living environment, malnutrition, lack of immunity, and exposure to tobacco smoke or indoor air pollutants, which may be adverse factors contributing to the occurrence of ARI in West Africa [60]. Exposure to air pollutants might act to increase the severity of respiratory infections and thereby increase the proportion of illnesses considered clinically to involve the lower respiratory tract, and even to increase morbidity and mortality. A number of air pollutants have been shown to adversely affect components of the defense mechanisms against infectious organisms [61]. The number of severe pneumonia cases in Sierra Leone was estimated to exceed 25,000 per year [62]. In the world, at least 2 billion people were exposed to the toxic effects of biomass fuel consumption, 1 billion were exposed to outdoor air pollution, and 1 billion were exposed to tobacco smoke [63]. In Sierra Leone, ARI prevalence was higher for children in homes with wood stoves compared with homes with charcoal stoves, but ARI prevalence for both types of fuels was higher compared with reported prevalence elsewhere [56]. Although the local government has been committed to garbage disposal, the environmental pollution caused by Garbage Mountain and long-term incineration of garbage cannot be ignored. In low-income countries, strengthening children's immunization programs and increasing the availability of pneumonia vaccines must be a priority.

A number of other common communicable diseases continued to affect the people of Sierra Leone [64]; $75 \%$ of patients with hepatitis B showed decompensated cirrhosis in the current study, and Gram negative bacteria were still the main pathogens of SBP. Cirrhosis with ascites was a chronic and severe disease, which was different from acute infectious diseases such as malaria, typhoid fever, diarrhea, and ARI. The ascites of liver cirrhosis was particularly noteworthy because patients with liver cirrhosis were prone to severe complications of ascites infection, causing fever, and acute progression leading to adverse consequences. In addition, even though the prevalence of hepatitis virus was rarely reported, a considerable number of patients in our hospital facility were patients with liver cirrhosis, and there was a lack of specialist hepatologists in the local area. Early diagnosis of bacterial infections in patients with hepatitis, especially those with decompensated cirrhosis, requires particular attention. Sierra Leone had one of the highest TB burdens per capita in the world, and its national HIV/AIDS prevalence rate was about $1.7 \%$ [65]. HIV infection increased risk for a number of febrile illnesses [66], and tuberculous encephalitis was found in the study. In order to respect the privacy of our patients, the research on HIV and TB infection was not included. National neglected tropical disease (NTD) is considered endemic in the country [29]. Sporadic cases including A. lumbricoides $1(1.4 \%)$ and filariasis $1(0.7 \%)$ were found. However, after the National NTD Master Plan 2016-2020 was officially launched, NTD has been significantly decreased in Sierra Leone [29].

This study indicated the importance of reliable diagnostic tests for febrile pathogens and provided the necessary information for RDT requirements. Malaria still threatens Sierra Leone; $22.5 \%$ were diagnosed with malaria infection. Limited by economic conditions, a considerable number of febrile patients in Africa bought medicine from pharmacies and adopted self-treatment methods; furthermore, asymptomatic patients could also lead to missed diagnosis. The implementation of malaria RDTs outside the hospital, such as pharmacy and chief tribe, could help reduce the misdiagnosis rate. For the diagnosis of typhoid, the baseline of antibody titer and the standardization were the key issues affecting the Widal test. Therefore, national standards could be established to improve the detection accuracy and effectively reduce the detection variation between regions. The etiology of patients with fever in Sierra Leone was closely related to the local geography, heredity, immune features, economic industry, air pollution, living habits, medical and health conditions. The local geography was suitable for the growth of Anopheles, which was the efficient local vector of $P$. falciparum. The genetic characteristics of Duffy negative blood group in the West African population were also 
related to the high prevalence of falciparum malaria. In endemic areas, frequent exposure to malaria and typhoid antigens leads to the immune features of asymptomatic parasitemia and increased baseline antibody titers. As a result of Sierra Leone being adjacent to the Atlantic Ocean, diarrhea pathogens were related to economic industries-fishery and aquatic products. Improper handling of human excreta could lead to rapid spread of diarrhea bacteria. In Sierra Leone, about 95\% of people depend on crude fuels, and the environmental pollution caused by incineration of garbage cannot be ignored and contributes to the occurrence of ARI. Strategies to improve medical and health conditions should also pay attention to some common chronic infectious diseases that cause fever, such as liver disease, tuberculosis, and so on. For empirical treatment, antimalarial treatment was still targeted at falciparum malaria in Sierra Leone. Furthermore, in this study, we found that Gram-negative bacteria, including S. typhi, S. flexneri, a variety of diarrhea bacteria and respiratory pathogens, were important pathogens of febrile diseases. In addition to $19.7 \%$ of patients infected with S. typhi, Gramnegative bacteria accounted for $92.9 \%$ of fecal culture-positive bacteria, $76.9 \%$ of sputum-culture positive bacteria, and all pathogens of spontaneous bacterial peritonitis. Therefore, antibiotics against Gram-negative bacteria contributed to the empirical treatment of febrile diseases.

This study has some limitations. First, we could not identify the etiological agents of fever in approximately $28.9 \%$ of the study population. A large number of data have shown that viral infection was an important pathogen in patients with ARI. Therefore, we did not carry out a large-scale screening of respiratory tractrelated viruses in this study $[6,67,68]$. In addition, considering the local conditions and the feasibility of popularization and application, we did not add blood and urine culture and drug sensitivity tests, which we believe will also improve the positive rate of etiological agents. Another febrile disease study conducted in Cameroon showed that the causative pathogen could not be identified in $30 \%$ of patients, and the spectra of febrile pathogens were significantly different from those in this study [69]. Secondly, there were insufficient data to assess the characteristics of coinfection in this study. As we known, the presence of viral-bacterial coinfection may well be a major trigger for the development of severe pneumonia [70]. Multiple infections were likely to lead to pathogen interaction and dynamics changes, which also suggested the need to expand the research object and carry out multicenter research.

\section{CONCLUSIONS}

The etiology of patients with fever in Sierra Leone was closely related to the local geography, heredity, immune features, economic industry, air pollution, living habits, medical and health conditions. Evidence of a wide spectrum of febrile pathogens emphasized the importance of a reliable diagnostic test for febrile pathogens and the necessary information for RDT requirements were provided from the study. The fever screening process of this study and its further simplification will help to carry out more effective diagnosis and empirical treatment of febrile illness in resource-limited areas in Sierra Leone. In addition, systematic and professional treatment of liver diseases should be promoted to reduce infection complications.

\section{ACKNOWLEDGEMENTS}

We wish to thank doctors, nurses, and other health workers from Joint Medical Unit and 34 Military Hospital, Freetown, Sierra Leone, for their assistance in study site coordination; We are very grateful to the staff at the Clinical Laboratory, 34 Military Hospital, Freetown, Sierra Leone. This paper is dedicated to Mr Zhenlin Wang in Beijing, China. We would like to express our highest respect and thanks to him, for this article is full of his great and selfless dedication. 
Funding. This study was supported by Military medical youth project, Chinese PLA General Hospital (Award Number QNC19033); and Youth development project for Military Medical Science and technology (Award Number 18QNP060); and China's national science and technology major special project (Award Number 2017ZX10304403004004). The funding agencies had no role in study design; collection, analysis and interpretation of data; or manuscript writing.

Authorship. All named authors meet the International Committee of Medical Journal Editors (ICMJE) criteria for authorship for this article, take responsibility for the integrity of the work as a whole, and have given their approval for this version to be published.

Authorship Contributions. HW: study design, funding acquisition, patient recruitment, specimen collection, data collection, statistical analysis and result interpretation, manuscript writing. QL: study design, statistical analysis, result interpretation, manuscript writing. WW, SS, MM, SC, KK and DIB: patient recruitment, specimen collection, data collection. JZ, NX, XH, YL, GN, YZ, GW, XL, YC, YY, $\mathrm{SB}$ : result interpretation, data arrangement, statistical analysis, manuscript writing. RQ: study design, specimen collection, result interpretation. All authors reviewed the manuscript, agreed to submission for publication, and approved the final version.

Disclosures. Han Wang, Jing Zhao, Na Xie, Wanxue Wang, Ruping Qi, Xiaogang Hao, Yan Liu, Stephen Sevalie, Guotao Niu, Yangli Zhang, $\mathrm{Ge} \mathrm{Wu}$, Xiaona Lv, Yuhao Chen, Yanfei Ye, Sheng Bi, Moses Moseray, Saidu Cellessy, Ksaidu Kalon, Dawud Ibrahim Baika and Qun Luo have nothing to disclose.

Compliance with Ethics Guidelines. The study was approved by the regional ethics committee in 34 Military Hospital, Republic of Sierra Leone Armed Forces (Deliberation JMU/ 1600). 34 Military Hospital approved this study to carry out etiological agents studies on human tissues, secretions, body fluids, and blood samples among febrile patients. The study was done according to the Helsinki Declaration. Informed consent was obtained from individuals (or their parents/guardians for children) for participation in this study.

Conflict of Interest. We have no competing interests to declare.

Data Availability. The datasets generated during and/or analyzed during the current study are available from the corresponding author on reasonable request.

Open Access. This article is licensed under a Creative Commons Attribution-NonCommercial 4.0 International License, which permits any non-commercial use, sharing, adaptation, distribution and reproduction in any medium or format, as long as you give appropriate credit to the original author(s) and the source, provide a link to the Creative Commons licence, and indicate if changes were made. The images or other third party material in this article are included in the article's Creative Commons licence, unless indicated otherwise in a credit line to the material. If material is not included in the article's Creative Commons licence and your intended use is not permitted by statutory regulation or exceeds the permitted use, you will need to obtain permission directly from the copyright holder. To view a copy of this licence, visit http://creativecommons.org/licenses/bync/4.0/.

\section{REFERENCES}

1. Government of Sierra Leone Ministry of Health and Sanitation. Sierra Leone Malaria Control Strategic Plan, 2016-2020. https://mohs.gov.sl/\#. Accessed 6 May 2020.

2. World Health Organization. World Malaria Report 2016. https://www.who.int/malaria/publications/ world-malaria-report-2016/report/en/. Accessed 25 Nov 2016

3. Government of Sierra Leone Ministry of Health and Sanitation. Health Information Bulletin 2020. https://mohs.gov.sl/\#. Accessed 20 Dec 2020. 
4. World Health Organization. World malaria report 2020: 20 years of global progress and challenges. https://www.who.int/teams/global-malariaprogramme. Accessed 30 Nov 2020.

5. Chappuis F, Alirol E, D'Acremont V, et al. Rapid diagnostic tests for non-malarial febrile illness in the tropics. Clin Microbiol Infect. 2013;19:422-31.

6. D'Acremont V, Kilowoko M, Kyungu E, et al. Beyond malaria-causes of fever in outpatient Tanzanian children. N Engl J Med. 2014;370:809-17.

7. Sokhna C, Gaye O, Doumbo O. Developing research in infectious and tropical diseases in Africa: the paradigm of Senegal. Clin Infect Dis. 2017;65: S64-9.

8. Prasad N, Murdoch DR, Reyburn H, Crump JA. Etiology of severe febrile illness in low- and middleincome countries: a systematic review. PLoS ONE. 2015;10:e0127962.

9. Baltzell K, Elfving K, Shakely D, et al. Febrile illness management in children under five years of age: a qualitative pilot study on primary health care workers' practices in Zanzibar. Malar J. 2013;12:37.

10. Gove S. Integrated management of childhood illness by outpatient health workers: technical basis and overview. The WHO Working Group on Guidelines for Integrated Management of the Sick Child. Bull World Health Organ. 1997;75(Suppl 1): 7-24.

11. Wenhong Zhang TL. Expert consensus on diagnosis and treatment of fever. Chin J Infect Dis. 2017;35: 641-55.

12. Nichter M. Acute respiratory infection. Introduction. Med Anthropol. 1994;15:319-34.

13. Bulla A, Hitze KL. Acute respiratory infections: a review. Bull World Health Organ. 1978;56:481-98.

14. Berman S. Epidemiology of acute respiratory infections in children of developing countries. Rev Infect Dis. 1991;13(Suppl 6):S454-462.

15. Campbell H. Acute respiratory infection: a global challenge. Arch Dis Child. 1995;73:281-3.

16. Grief SN. Upper respiratory infections. Prim Care. 2013;40:757-70.

17. Saubolle MAMP. Laboratory diagnosis of community-acquired lower respiratory tract infection. Infect Dis Clin N Am. 2001;15:1025-45.

18. Regamey N, Kaiser L, Roiha HL, et al. Viral etiology of acute respiratory infections with cough in infancy: a community-based birth cohort study. Pediatr Infect Dis J. 2008;27:100-5.

19. Cordey S, Thomas Y, Cherpillod P, et al. Simultaneous detection of parainfluenza viruses 1 and 3 by real-time reverse transcription-polymerase chain reaction. J Virol Methods. 2009;156:166-8.

20. Jorgensen JHMAP, Carroll KC, et al. Manual of clinical microbiology, 11th Edition. Washington, DC: ASM Press; 2015.

21. Cheesbrough M. District laboratory practice in tropical countries. 2nd ed. Cambridge: Cambridge University Press; 2005.

22. Rahman MA. Antimicrobial resistance patterns of Salmonella Typhi isolated from stool culture. Chattagram Maa-O-Shishu Hospital Medical College Journal. 2015;14:26-30.

23. Romney M, Humphries AJL. Practical guidance for clinical microbiology laboratories: diagnosis of bacterial gastroenteritis. Clin Microbiol Rev. $2015 ; 28: 3-31$.

24. Yue P, Zhou M, Zhang L, et al. Clinical performance of BD Kiestra InoqulA automated system in a Chinese tertiary hospital. Infect Drug Resist. 2020;13: 941-7.

25. Wiest R, Krag A, Gerbes A. Spontaneous bacterial peritonitis: recent guidelines and beyond. Gut. 2012;61:297-310.

26. Marais S, Thwaites G, Schoeman JF, et al. Tuberculous meningitis: a uniform case definition for use in clinical research. Lancet Infect Dis. 2010;10:803-12.

27. Organization WH. Global Health Estimates: Life expectancy and leading causes of death and disability. World Health Data Platform 2019.

28. Takem EN, Roca A, Cunnington A. The association between malaria and non-typhoid Salmonella bacteraemia in children in sub-Saharan Africa: a literature review. Malar J. 2014;13:400.

29. World Health Organization. Year in focus Sierra Leone annual report 2017. Annual Report WHO. Sierra Leone Country Office: World Health Organization; 2017.

30. Sigaúque B, Roca A, Mandomando I, et al. Community-acquired bacteremia among children admitted to a rural hospital in Mozambique. Pediatr Infect Dis J. 2009;28:108-13.

31. Enwere G, Biney E, Cheung YB, et al. Epidemiologic and clinical characteristics of community-acquired invasive bacterial infections in children aged 2-29 
months in The Gambia. Pediatr Infect Dis J. 2006;25:700-5.

32. Leski TA, Taitt CR, Swaray AG, et al. Use of real-time multiplex PCR, malaria rapid diagnostic test and microscopy to investigate the prevalence of Plasmodium species among febrile hospital patients in Sierra Leone. Malar J. 2020;19:84.

33. Odikamnoro IMI, Okoh FN, Ebiriekwe SC, et al. Incidence of malaria/typhoid co-infection among adult population in Unwana community, Afikpo north local government area, Ebonyi state, Southeastern Nigeria. Afr J Infect Dis. 2017;12:33-8.

34. Barnish G, Maude GH, Bockarie MJ, et al. Malaria in a rural area of Sierra Leone. II. Parasitological and related results from pre- and post-rains clinical surveys. Ann Trop Med Parasitol. 1993;87:137-48.

35. Miller L, Mason S, Dvorak J, et al. Erythrocyte receptors for (Plasmodium knowlesi) malaria: Duffy blood group determinants. Science. 1975;189: 561-3.

36. Birhanie BTM, Ferede G, Endris M, et al. Malaria, typhoid fever, and their coinfection among febrile patients at a rural health center in northwest Ethiopia: a cross-sectional study. Adv Med. 2014;2014:531074.

37. Jalloh A, Jalloh M, Matsuoka H. T-cell epitope polymorphisms of the Plasmodium falciparum circumsporozoite protein among field isolates from Sierra Leone: age-dependent haplotype distribution? Malar J. 2009;8:120.

38. Barnish G, Maude GH, Bockarie MJ, et al. The epidemiology of malaria in southern Sierra Leone. Parassitologia. 1993;35(Suppl):1-4.

39. Pitzer VE, Meiring J, Martineau FP, et al. The invisible burden: diagnosing and combatting typhoid fever in Asia and Africa. Clin Infect Dis. 2019;69:s395-401.

40. Sundufu AJJMS, Foday IK. Role of co-infection with malaria parasites and salmonella typhoid in Bo City, Southern Sierra Leone. Public Health Res. 2013;2:204-7.

41. Kargbo MS, Massaquoi LD, Samura SK, et al. The relative prevalence of typhoid and malaria in febrile patients in Freetown, Sierra Leone. Open J Prev Med. 2014;4:338-46.

42. Ley B, Mtove G, Thriemer K, et al. Evaluation of the Widal tube agglutination test for the diagnosis of typhoid fever among children admitted to a rural hospital in Tanzania and a comparison with previous studies. BMC Infect Dis. 2010;10:180.
43. Parry CM, Hoa NTT, Diep TS, et al. Value of a singletube Widal test in diagnosis of typhoid fever in Vietnam. J Clin Microbiol. 1999;37:2882-6.

44. Andualem G, Abebe T, Kebede N, et al. A comparative study of Widal test with blood culture in the diagnosis of typhoid fever in febrile patients. BMC Res Notes. 2014;7:653.

45. Youssef FGDAS, Kabeil SS, Parker TM. A comparative study of blood culture and antibody response with the duration of illness in diagnosis of typhoid fever Australian. J Basic Appl Sci. 2010;4:609-14.

46. Chew SKCM, Lim YS, Monteiro EH. Diagnostic value of the Widal test for typhoid fever in Singapore. J Trop Med Hyg. 1992;95:288-91.

47. Hoffman SL, Flanigan TP, Klaucke D, et al. The Widal slide agglutination test, a valuable rapid diagnostic test in typhoid fever patients at the Infectious Diseases Hospital of Jakarta. Am J Epidemiol. 1986;123:869-75.

48. Pokhrel BM, Karmacharya R, Mishra SK, Koirala J. Distribution of antibody titer against Salmonella enterica among healthy individuals in nepal. Ann Clin Microbiol Antimicrob. 2009;8:1.

49. Olopoenia ALK. Widal agglutination test - 100 years later: still plagued by controversy. Postgrad Med J. 2000;76:80-4.

50. Sansone PMSS, Hennekens $\mathrm{CH}$ Jr. High titer Widal reaction. JAMA. 1972;220:1615-6.

51. Nsutebu EF, Martins P, Adiogo D. Prevalence of typhoid fever in febrile patients with symptoms clinically compatible with typhoid fever in Cameroon. Trop Med Int Health. 2003;8:575-8.

52. Ammah A, Nkuo-Akenji T, Ndip R, Deas JE. An update on concurrent malaria and typhoid fever in Cameroon. Trans R Soc Trop Med Hyg. 1999;93: 127-9.

53. Duthie R, French GL. Comparison of methods for the diagnosis of typhoid fever. J Clin Pathol. 1990;43:863-5.

54. Ohanu ME, Maduakor U, Onodugo OD, et al. Typhoid fever among febrile Nigerian patients: prevalence, diagnostic performance of the Widal test and antibiotic multi-drug resistance. Malawi Med J. 2019;31:184-92.

55. Kullin B, Meggersee R, D'Alton J, et al. Prevalence of gastrointestinal pathogenic bacteria in patients with diarrhoea attending Groote Schuur Hospital, Cape Town, South Africa. S Afr Med J. 2015;105: 121-5. 
56. Taylor ET, Nakai S. Prevalence of acute respiratory infections in women and children in western Sierra Leone due to smoke from wood and charcoal stoves. Int J Environ Res Public Health. 2012;9: 2252-65.

57. Woolley KE, Bagambe T, Singh A, Avis WR, et al. Investigating the association between wood and charcoal domestic cooking, respiratory symptoms and acute respiratory infections among children aged under 5 years in Uganda: a cross-sectional analysis of the 2016 demographic and health survey. Int J Environ Res Public Health. 2020;17:3974.

58. Von Nguyen DNS, Lam E, Ayers T, et al. Cholera epidemic associated with consumption of unsafe drinking water and street-vended water-Eastern Freetown, Sierra Leone, 2012. Am J Trop Med Hyg. 2014;90:518-23.

59. Weber MW, Dackour R, Usen S, et al. The clinical spectrum of respiratory syncytial virus disease in The Gambia. Pediatr Infect Dis J. 1998;17:224-30.

60. Almirall J, Serra-Prat M, Bolíbar I, Balasso V. Risk factors for community-acquired pneumonia in adults: a systematic review of observational studies. Respiration. 2017;94:299-311.

61. Woolley KE, Bagambe T, Singh A, et al. Investigating the association between wood and charcoal domestic cooking, respiratory symptoms and acute respiratory infections among children aged under 5 years in Uganda: a cross-sectional analysis of the 2016 demographic and health survey. Int J Environ Res Public Health. 2020;17:3974.

62. Ministry of Public Health and Sanitation of Republic of Sierra Leone. INTEGRATED DISEASE SURVEILLANCE AND RESPONSE (IDSR) Sierra Leone Epidemiological Report 2016. https://www. afro.who.int/publications/sierra-leone-epidemiolo gical-report-2016. Accessed 01 Jan 2017.

63. Forum of International Respiratory, European Respiratory Society. Respiratory diseases in the world
Realities of Today-Opportunities Tomorrow. https://www.ersnet.org/pdf/publications/firs-worldreport.pdf. Accessed 06 Oct 2013.

64. O'Shea MK, Clay KA, Craig DG, et al. Diagnosis of febrile illnesses other than Ebola virus disease at an Ebola treatment unit in Sierra Leone. Clin Infect Dis. 2015;61:795-8.

65. Yendewa GA, Poveda E, Yendewa SA, et al. HIV/ AIDS in Sierra Leone: characterizing the hidden epidemic. AIDS Rev. 2018;20:104-13.

66. Mehri A, Alimohamadi Y, Mohammadi M, et al. Trend of HIV and tuberculosis co-epidemics in different regions of World Health Organization during 2003-2017. HIV AIDS Rev. 2020;19:167-71.

67. Selvaraju SB, Kovac M, Dickson LM, et al. Molecular epidemiology and clinical presentation of human adenovirus infections in Kansas City children. J Clin Virol. 2011;51:126-31.

68. Brodzinski H, Ruddy RM. Review of new and newly discovered respiratory tract viruses in children. Pediatr Emerg Care. 2009;25:352-60.

69. Achonduh-Atijegbe OA, Mfuh KO, Mbange AHE, et al. Prevalence of malaria, typhoid, toxoplasmosis and rubella among febrile children in Cameroon. BMC Infect Dis. 2016;16:658.

70. Cevey-Macherel M, Galetto-Lacour A, Gervaix A, et al. Etiology of community-acquired pneumonia in hospitalized children based on WHO clinical guidelines. Eur J Pediatr. 2009;168:1429-36.

\section{Publisher's Note}

Springer Nature remains neutral with regard to jurisdictional claims in published maps and institutional affiliations. 